\title{
Hand-Held Diode Laser for On-Site Analysis Using Transportable Mass Spectrometry
}

\author{
Ane Arrizabalaga-Larrañaga, Michel W. F. Nielen, and Marco H. Blokland*
}

Cite This: Anal. Chem. 2021, 93, 8122-8127

Read Online

ABSTRACT: A hand-held laser diode thermal desorption electrospray ionization (LDTD-ESI) mass spectrometry (MS) method was developed for rapid screening of illegal substances in solid samples. To achieve that, a simple, inexpensive, battery-powered surgical laser diode at $940 \mathrm{~nm}$ was employed to ablate the solid samples. The potential of using a black polytetrafluoroethylene substrate to enhance the analytes' desorption to the gas phase was investigated and demonstrated. Among the optimized ESI parameters, the solvent (methanol/water, 50:50,v/v) and the flow rate $\left(50 \mu \mathrm{L} \mathrm{h}^{-1}\right)$ were critical to obtain the best sensitivity. The applicability was demonstrated for the rapid identification of selective androgen receptor modulators (SARMs) in pills and powders based on accurate

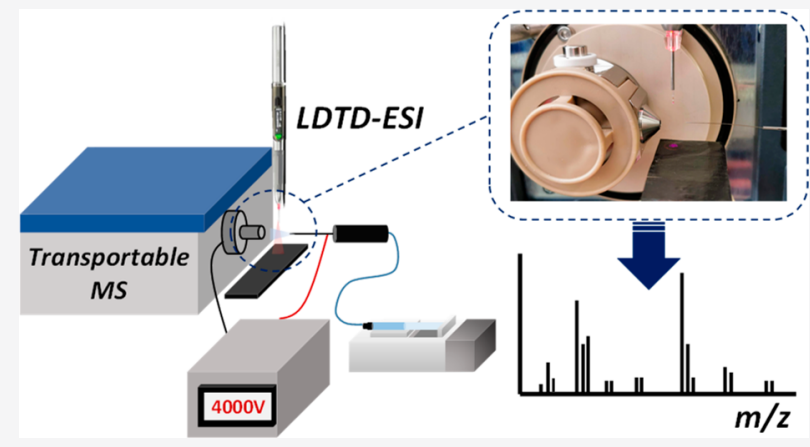
mass measurements by time-of-flight MS. Also, the hand-held LDTD-ESI was combined with a transportable single quadrupole MS. The same SARMs samples were analyzed, and identifications were based on in-source cone voltage fragmentation patterns observed. These initial results demonstrate the applicability of the developed simplified LDTD-ESI MS method for future on-site testing of organic compounds in solid samples.

\section{INTRODUCTION}

Rapid and easy-to-perform methods for on-site analysis have a bright future in forensic, environmental, and food analysis. ${ }^{1,2}$ On-site prescreening of samples for target compounds will reduce the number of suspicious samples transported to the laboratory for analysis and make control and monitoring systems more effective. Examples of successful prescreening are by the use of portable and smartphone-based sensors such as near-infrared (NIR) scanners with chemometrics for a comprehensive characterization of the chemical composition of milk tanks, ${ }^{3}$ portable hyphenated photonics sensors for detecting food fraud in extra virgin olive oil, ${ }^{4}$ and oral fluid drug tests in recreational contexts. ${ }^{5}$ However, it is impossible to differentiate between molecules of similar physicochemical properties and structures by these spectrometric techniques since the obtained spectra are not that specific. Lateral flow immunoassays (LFIAs), on the other hand, rely on biorecognition and are employed to identify specific substances such as antibiotic residues, mycotoxins, and multiplex allergens in milk, cereals, and nuts, respectively. ${ }^{6-8}$ But LFIAs cannot identify the substance because, depending on the crossreactivity profile, similar compounds may yield a signal as well. On-site mass spectrometry (MS) could solve these drawbacks because of a much more specific spectrum, leading to direct identification of compounds. 9

Ambient ionization mass spectrometry (AIMS) techniques have been developed, offering simplified sample preparation and sample introduction protocols prior to analysis. For instance, desorption electrospray ionization (DESI), ${ }^{10}$ direct analysis in real-time (DART), ${ }^{11}$ low temperature plasma (LTP), ${ }^{12}$ laser ablation electrospray ionization (LAESI), ${ }^{13}$ paper spray (PS), ${ }^{14}$ and atmospheric solids analysis probe (ASAP) $)^{15}$ are the main AIMS techniques employed. So far, only a few studies reported ambient ionization of food contaminants and drug analysis with a (trans)portable MS system. ${ }^{16-19}$ For on-site MS, vulnerable laser setups and heavy gas cylinders should be avoided, whereas a small footprint, low weight, and low power consumption are required. ${ }^{20}$ Also, the technique should offer robustness and acceptable analytical performance for screening. The sample cleanup and introduction into a fieldable mass-spectrometer should be fast, and also, nonexpert users should be capable of operating them. Generally, gaseous samples can be introduced straightforwardly, whereas liquid samples usually proceed following dilution or liquid-liquid extraction of the targeted compounds. In contrast, the analysis of solid samples requires more laborious extraction procedures and cleanup steps. Regardless, laser-based techniques are well established for

Received: March 11, 2021

Accepted: May 18, 2021

Published: June 2, 2021 
sampling solid materials at atmospheric pressure, and mainly, ultraviolet or infrared lasers have been used to desorb/ablate the analyte from a solid sample surface. ${ }^{21,22}$ Most of the commercially available lasers have a fixed and precisely aligned setup, hindering their applicability for on-site analysis. Recently, Yung et al. ${ }^{23}$ reported the use of a hand-held diode laser in combination with atmospheric pressure photoionization (APPI) for the solid sampling of plant and microbial communities. This laser was developed for dental surgical applications as a source of infrared radiation $(940 \mathrm{~nm})$ and could be the breakthrough for analyzing solid samples in the field, enabling time and cost savings of analyses.

In this study, the potential of a hand-held diode laser coupled to a high-resolution quadrupole time-of flight (QTOF) MS and a transportable quadrupole MS for on-site fast screening of selective androgen receptor modulators (SARMs) in solid samples is demonstrated. For this purpose, the hand-held laser setup was optimized, and the most critical working parameters were evaluated and are discussed.

\section{EXPERIMENTAL SECTION}

Chemicals. Methanol, acetonitrile, and water of UHPLCMS purity grade, as well as formic acid, ammonium formate, ammonium acetate, and ammonium fluoride, were supplied from Actu-All Chemicals (Oss, The Netherlands). Ammonia solution 25\% and acetic acid (glacial) 100\% were purchased from Merck (Darmstadt, Germany). Microscope glass slides $(76 \mathrm{~mm} \times 26 \mathrm{~mm})$ were purchased from Thermo Fisher Scientific (Waltham, MA, USA), and microscope glass slides $(76 \mathrm{~mm} \times 26 \mathrm{~mm})$ having 66 white polytetrafluoroethylene (PTFE) printed spots $7.1 \mathrm{~mm}^{2}$ were purchased from Waters Corporation (Milford, MA, USA). Nostik baking foil for black PTFE slides was purchased from a local supermarket. Six selective androgen receptor modulators (Figure S1) were used as the model system for the optimization of LDTD-ESI parameters. Analytical standards of andarine, ibutamoren, ligandrol, ostarine, stenabolic, and testolone were purchased from Selleck Chemicals (Houston, TX, USA). Individual stock solutions $\left(1000 \mathrm{mg} \mathrm{L}^{-1}\right)$ were prepared in ethanol and stored at $-80{ }^{\circ} \mathrm{C}$. Intermediate individual solutions $\left(10 \mathrm{mg} \mathrm{L}^{-1}\right)$ and a standard mixture solution $\left(1 \mathrm{mg} \mathrm{L}^{-1}\right)$ containing all target compounds were prepared monthly from stock standard solutions by appropriate dilution in acetonitrile:water (50:50, $v / v)$. All these standard solutions were stored at $4{ }^{\circ} \mathrm{C}$ until their use. Only $2 \mu \mathrm{L}$ of a standard solution $\left(1 \mathrm{mg} \mathrm{L}^{-1}\right)$ in acetonitrile:water $(50: 50, v / v)$ was spotted on the substrate slides for all compounds.

Instrumentation. A hand-held diode laser $(100 \mathrm{~g}, 21 \mathrm{~cm}$ long) designed for dental surgical applications (iLase, Biolase Tech, Irvine, CA, USA) operating at $940 \mathrm{~nm}$ for sample desorption and equipped with $1 \mathrm{~h}$ lifetime rechargeable batteries was used in this study. The laser beam was directed to the ablation zone via a disposable $400 \mu \mathrm{m}$ fiber optic tip. The peak power optical output was set at $3 \mathrm{~W}$ with an average power density of $1 \mathrm{~W}$. The device was operated in pseudo-CW mode with a pulse length of $10 \mathrm{~ms}$ in which the pulse is $0.1 \mathrm{~ms}$ on followed by $0.2 \mathrm{~ms}$ off. The ablation spot size was approximately $1.5 \mathrm{~mm}^{2}$. The mass spectrometric analyses were carried out using a Bruker Impact II QTOF mass spectrometer (Bruker, Billerica, MA). The electrospray (ESI) source was removed, and an ESI emitter supplied by Waters (Milford, Massachusetts, USA) was placed in front of the MS-inlet. Solvent to the ESI emitter was delivered using an external KD
Scientific syringe pump purchased from Antec Leyden (Zoeterwoude, The Netherlands). The source capillary voltage was 4000 and $3500 \mathrm{~V}$ in positive ion and negative ion modes, respectively, whereas the end plate offset was $500 \mathrm{~V}$. The source drying gas and temperature were set at $0.1 \mathrm{~L} \mathrm{~min}^{-1}$ and $150{ }^{\circ} \mathrm{C}$, respectively. The full scan mass spectra were obtained over a mass range of $\mathrm{m} / z 100-600$, at a resolution of 60,000 FWHM. A solution of methanol:water $(50: 50, v / v)$ with $0.1 \%$ formic acid was used as electrospray solvent in positive ion mode, whereas in negative ion mode, a solution of methanol:water $(50: 50, v / v)$ with $0.1 \%$ ammonia was employed at a constant flow rate of $50 \mu \mathrm{L} \mathrm{h}^{-1}$. The QTOF was calibrated every day in both positive ion and negative ion modes using 10 $\mathrm{mM}$ sodium formate. Compass Data Analysis v4.4 software (Bruker) was used to control the instrument setup and acquire and process the MS data.

The LDTD-ESI setup was also combined with a transportable single quadrupole model Acquity QDa detector MS system (Waters Corporation, Manchester, U.K.). Prior to use, the instrument was slightly modified by taking out the ESI source and changing the instrument settings, according to Devereaux et al. ${ }^{24}$ The mass spectral data were acquired in full scan in positive ion mode. A source capillary voltage of $4000 \mathrm{~V}$ was applied to the ESI emitter, whereas the cone voltage was held at $30 \mathrm{~V}$ and the source temperature at $150{ }^{\circ} \mathrm{C}$. Instrument control and analysis of MS data were carried out using Mass Lynx v4.1 software (Waters). The home-built LDTD-ESI source setup shown in Figure 1 is based on a nano-ESI emitter to which at $90^{\circ}$ a hand-held laser is positioned by hand. A distance of $5 \mathrm{~mm}$ was set between the mass spectrometer inlet

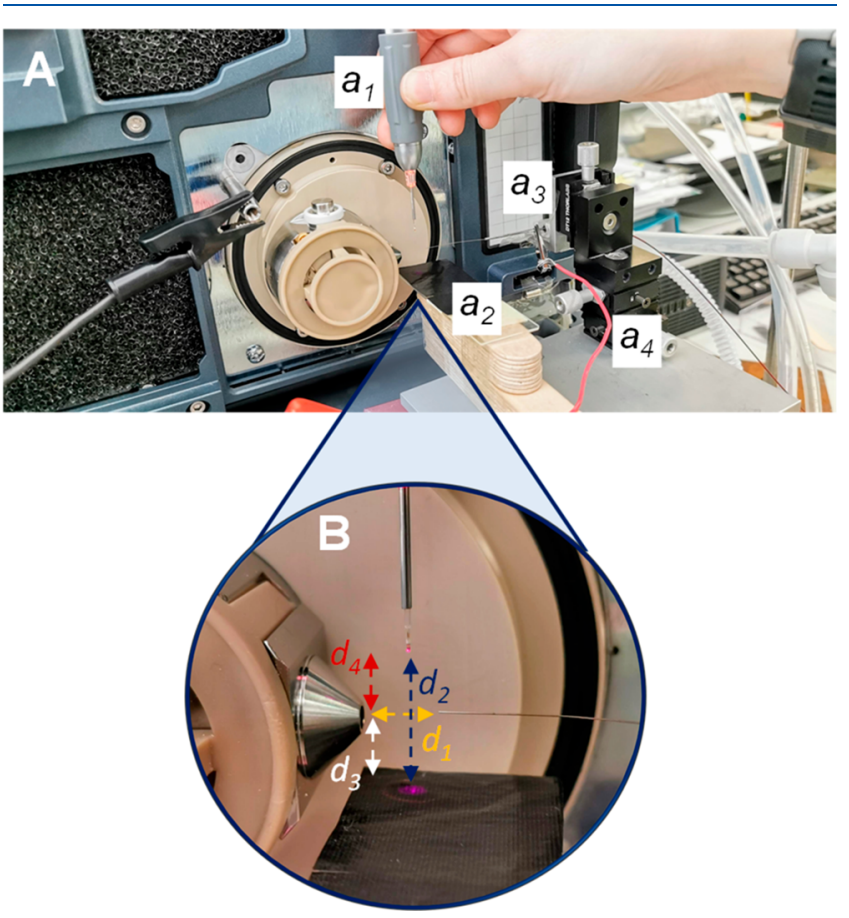

Figure 1. (A) Transportable single quadrupole MS setup: $\left(a_{1}\right)$ handheld battery-powered hand-held $940 \mathrm{~nm}$ diode laser, $\left(\mathrm{a}_{2}\right)$ black PFTE substrate, $\left(a_{3}\right)$ nano-ESI emitter, and $\left(a_{4}\right)$ voltage supply. (B) Exploded view of the MS entrance, tip of the laser, and ESI emitter: $\left(d_{1}\right)$ distance between the mass spectrometer inlet and the spray tip, $\left(d_{2}\right)$ distance from the portable laser tip to the sample surface, $\left(d_{3}\right)$ distance from the sample surface to the mass spectrometer, and $\left(\mathrm{d}_{4}\right)$ distance from the laser tip to the sample cone. 
and the spray tip $\left(d_{1}\right)$ at an angle of $180^{\circ}$. Samples were deposited onto the sample stage. The distance from the portable laser tip to the sample surface $\left(d_{2}\right)$ was set as $15 \mathrm{~mm}$, with the distance from the sample surface to the mass spectrometer $\left(d_{3}\right)$ set as $10 \mathrm{~mm}$ and the distance from the laser tip to the sample cone $\left(d_{4}\right)$ set as $5 \mathrm{~mm}$.

Samples. Samples were from in-house stock and were previously checked to determine if they contained any SARMs. In total, 10 samples were analyzed, from which four were pills and six powder samples that could be used as an illegal growth promotor in sports doping or animal husbandry. Direct LDTD-ESI analysis of the samples was performed without any sample pretreatment. Pills were analyzed directly from both the outside and inside parts and as powders by placing them directly onto the black PTFE substrate.

\section{RESULTS AND DISCUSSION}

Substrate Characterization. The hand-held laser employed in this work is a dental soft tissue laser designed for surgical applications. It uses a solid-state laser diode as a source of invisible infrared radiation $(940 \mathrm{~nm})$, and the energy is delivered to the sample stage via a fiber optic tip assembly. The energy generated by the laser's infrared radiation may be converted into heat on the surface of the sample stage, causing analytes to be ablated by sublimation, flash evaporation, or even a pyrolysis mechanism. Subsequently, the ablated sample material is extracted by the charged droplets from the nanoESI emitter. The sample surface/substrate is expected to have an important role in the desorption/ablation process. A wavelength of $2940 \mathrm{~nm}$ has been used by LAESI, whereby vibrational transitions from the $\mathrm{O}-\mathrm{H}$ bond of the water matrix or the analyte itself are activated. ${ }^{13,25,26}$ However, at $940 \mathrm{~nm}$, the $\mathrm{O}-\mathrm{H}$ bond is not absorbing the IR radiation, and thus, the ablation must be supported by the type of material on the sample stage. The solid surface characteristics, including its chemical composition and texture, severely affects the energy transfer processes and, therefore, the desorption efficiency. In this study, three different surfaces were tested as substrates to ablate and analyze the target compounds: glass, paper, and PTFE (white and black). A low irreproducible signal was observed using glass slides because the analytes' distribution on the surface was not focused following solution deposition. No signal of the analytes was obtained from paper slides, probably because the paper tends to absorb the spotted solution. From the PTFE materials, the black PTFE surface showed the highest signal intensity as well as the best signal stability.

The substrate's color is important since it influences the absorption of the emitted IR radiation and indirectly heats the sample. PTFE surfaces with both white and black colors were evaluated.

As can be observed in Figure 2a, the base peak chronogram (BPC) of a blank solvent spot on the white PTFE substrate is 10 times lower than on the black PTFE (Figure 2b). White and black PTFE substrates were also tested on two compounds of interest; Figure $2 \mathrm{c}$ and $\mathrm{d}$ depict the extracted ion chronograms (EIC) of ostarine $(\mathrm{m} / z 388.091)$ and andarine $(\mathrm{m} / z$ 440.107) for both colors. The targeted compounds' signal intensities when using the black PTFE are 100 times higher compared to the white PFTE. Being a black object, all wavelengths of light, including the $940 \mathrm{~nm}$ infrared wavelength of our diode laser, are absorbed and converted into heat. Hence, the PTFE film heats up rapidly, resulting in the analytes' sublimation and transfer to the gas phase followed by post-ESI ionization. a)
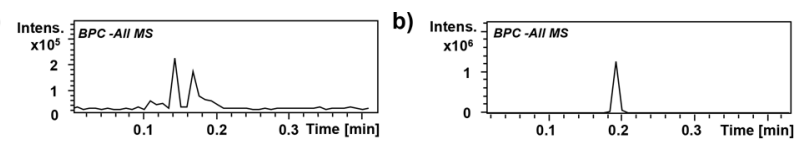

c)
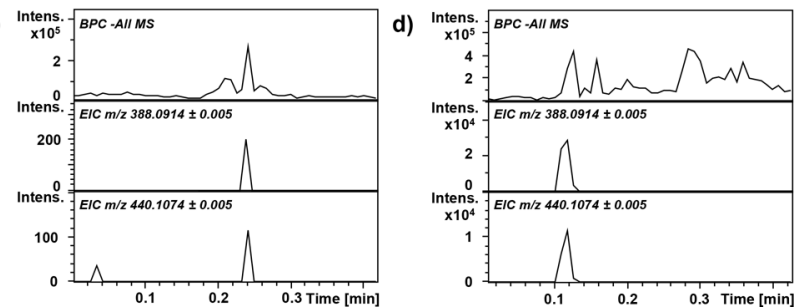

Figure 2. Chronograms obtained by LDTD-ESI-HRMS: base peak chronogram of a blank with (a) white PTFE substrate and (b) black PTFE substrate and extracted ion chronogram of SARMs ostarine and andarine spotted onto (c) white PTFE substrate and (d) black PTFE substrate.

Therefore, we propose to classify the ionization mechanism as LDTD-ESI.

LDTD-ESI-HRMS. The LDTD-ESI ionization process was optimized with respect to the (i) electrospray process, (ii) laser process, and (iii) geometrical LDTD-ESI parameters. Six selective androgen receptor modulators were used as model compounds to optimize the LDTD-ESI-HRMS working conditions. Standard solutions of the target compounds (1 $\mathrm{mg} \mathrm{L}^{-1}$ ) were deposited on black PTFE surfaces, and their full scan mass spectra were recorded using both positive ion and negative ion modes. Stenabolic, ligandrol, ibutamoren, and testolone were ionized in positive ion mode showing the protonated molecules $[\mathrm{M}+\mathrm{H}]^{+}$, whereas ostarine and andarine were ionized in negative ion mode yielding the deprotonated molecule $[\mathrm{M}-\mathrm{H}]^{-}$(Figure S3a, b). In the mass spectra of standards, apart from the ions observed in the lower mass region owing to the black PTFE substrate, no significant thermal fragmentation or adduct formation from the SARM analytes was observed in any case.

Electrospray solvent composition has a crucial effect on both the ionization and transfer of the analytes to the mass spectrometer. $^{27,28}$ For instance, Nemes et al. ${ }^{13}$ proposed $50 \%$ methanol acidified with $0.1 \%$ acetic acid to promote the ionization of different drugs in positive LDTD-ESI. Therefore, different solvent mixtures of methanol/water and acetonitrile/ water and the addition of modifiers such as formic acid or acetic acid to promote the protonation of target compounds in the positive ion mode and ammonia or ammonium salts $\left(\mathrm{NH}_{4} \mathrm{Fo}, \mathrm{NH}_{4} \mathrm{Ac}\right.$, and $\left.\mathrm{NH}_{4} \mathrm{~F}\right)$ to deprotonate in the negative ion mode were evaluated. The solvent composition influences the analytes ionization efficiency, and the highest signal intensity was achieved using methanol/water with $0.1 \%$ of ammonia (Figure 3a). This could be attributed to the higher basicity compared to the ammonium formate, ammonium acetate, and ammonium fluoride salts. The organic solvent composition was also compared between methanol/water and acetonitrile/water mixtures, and the highest signal intensities were obtained with methanol/water mixtures, probably due to the higher solubility of the analytes in this composition, which improves the transfer efficiency of the ablated analytes. The organic solvent percentage on the ion signal intensity was also studied using different methanol/water mixtures (Figure 3b). Both compounds showed similar behavior, and the ion signal was the highest when the organic solvent content was equal to the water content. Thus, the optimal solvent composition 
a)

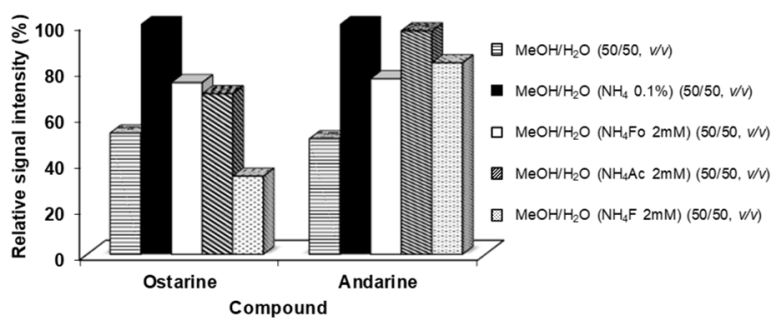

b)

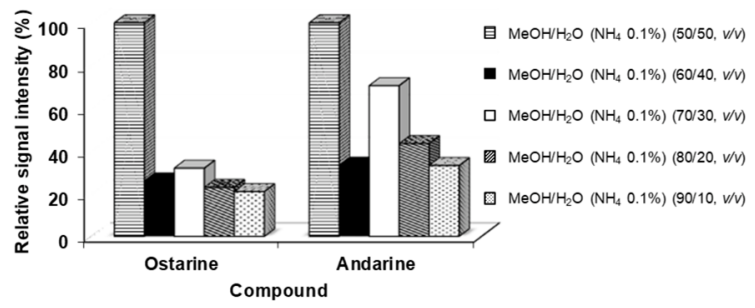

Figure 3. Effect of the LDTD-ESI solvent additive (a) and the percentage of methanol in the LDTD-ESI-HRMS signal for some representative SARMs (b).

selected for negative ion mode studies was $0.1 \%$ ammonia in methanol/water $(50: 50, v / v)$. For the positive ion mode, methanol:water $(50: 50, v / v)$ with $0.1 \%$ of formic acid showed the best efficiency to protonate the target compounds. The solvent flow rate also affects the ionization of the analytes, and thus, it was optimized using the previously selected solvent composition. The flow rate was tested from 30 to $500 \mu \mathrm{L} \mathrm{h}^{-1}$, and it was observed that when using a flow rate of $50 \mu \mathrm{L} \mathrm{h}^{-1}$ the signal of the targeted compounds was the highest (Figure S4). As the flow rate increased, the signal was decreasing until the point that at $500 \mu \mathrm{L} \mathrm{h}^{-1}$ the signal intensity of the analytes was negligible, probably due to the bigger droplet formation. Thus, a solvent flow rate of $50 \mu \mathrm{L} \mathrm{h}^{-1}$ was chosen as the optimal working condition.

To optimize the laser parameters, $0.1 \%$ ammonia in methanol/water $(50: 50, v / v)$ at $50 \mu \mathrm{L} \mathrm{h}^{-1}$ was used as the ESI solvent. The 11 preprogrammed settings available in the hand-held laser (Table S1) that vary in peak power and pulse models were evaluated. The power peak of the programs differs from 1.6 to $3.0 \mathrm{~W}$, whereas the pulse models can be CP1, $(0.1$ $\mathrm{ms}$ on, followed by $0.2 \mathrm{~ms}$ off), CP2 (1.0 ms on followed by $1.0 \mathrm{~ms}$ off), or CW (continuous wave output). Among the programs with a pulse model of $\mathrm{CP} 2$, the highest signal intensity of the target compounds was obtained when the peak power was $2 \mathrm{~W}$ (program 2). A considerable improvement in the analyte's response was by changing the pulse length to CP1 and the power to $3 \mathrm{~W}$. This program (program 1) was selected as the optimal preprogrammed setting for the analysis of these compounds. The LDTD-ESI geometrical parameters were optimized by varying the ESI emitter's distance to the inlet and the portable laser position. Therefore, the spray tip's distance to the mass spectrometer $\left(d_{1}\right)$ was varied from 5 to $10 \mathrm{~mm}$. The highest signal responses in all cases were observed to be at a distance of $5 \mathrm{~mm}$. The distance from the portable laser tip to the sample surface $\left(d_{2}\right)$ and mass spectrometer inlet $\left(d_{3}\right)$ was optimized. The effect of $d_{2}$ and $d_{3}$ on LDTD-ESI was evaluated by modifying the distances $(5-20 \mathrm{~mm})$ and $(10-20 \mathrm{~mm})$, respectively. The highest signal intensity was obtained when the laser tip was $15 \mathrm{~mm}\left(180^{\circ}\right)$ from the sample surface and 10 $\mathrm{mm}\left(90^{\circ}\right)$ from the mass spectrometer.

LDTD-ESI-HRMS Screening of Samples. Selective androgen receptor modulators (SARMs) are nonsteroidal compounds commonly described as drugs of abuse in human and animal sports, with the potential for misuse as growth promoters in animal-based food production. ${ }^{29}$ As a response to the potential illicit application of SARMs in sports doping or in food production systems, the applicability of the developed LDTD-ESI-HRMS method was evaluated by analyzing 10 samples. The powders of the samples were directly screened by putting a small amount on the sample stage. The samples were directly ablated without any pretreatment using the hand-held laser. The obtained $\mathrm{m} / \mathrm{z}$ values were assigned to SARMs (within $5 \mathrm{ppm}$ of the theoretical mass). Additionally, since some of the samples were pills, they were also analyzed by applying the laser directly on the outside and inside parts. No signal was observed in the LDTD-ESI-HRMS spectrum for either the outside or inside. However, identifying SARMs in these samples was possible when the pills were powdered, and the powder was put on the sample stage (Figure S5). This experiment again emphasizes the necessity of the black PFTE substrate to desorb the material. The power alone from the laser is not enough for the desorption of compounds directly from the pills themselves. The analyzed samples and the identified selective androgen receptor modulators are listed in Table S2. Figure 4 shows the LDTD-ESI-HRMS spectrum of two positive samples where (a) stenabolic and (b) ostarine were identified.
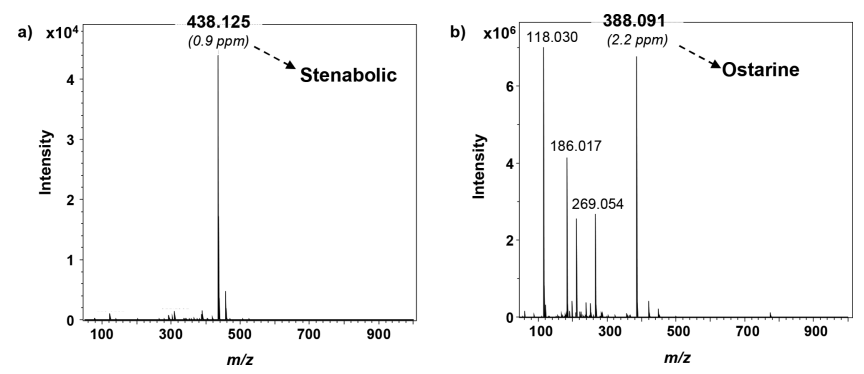

Figure 4. LDTD-ESI-HRMS full-scan spectrum obtained from samples 1 (a) and 10 (b), containing stenabolic and ostarine, respectively.

Proof of Principle of Hand-Held LDTD-ESI on a Transportable MS System. The developed hand-held LDTD-ESI source was also coupled to a transportable single quadrupole mass spectrometer. This MS system can be easily transported and is operational within $10 \mathrm{~min}$ after plugging it into a power source ${ }^{30}$ (see Figure 1 for the experimental setup). Four of the previously analyzed samples were ablated, similar to the experiments on the HRMS. In these experiments, the data acquisition was performed in full scan mode $(\mathrm{m} / \mathrm{z}$ 120-500). For identification, in-source fragmentation was applied by increasing the cone voltage. The respective standards were individually analyzed, and the tentative fragment ions were assigned (Table 1).

The samples were analyzed in full scan mode applying $30 \mathrm{~V}$ cone voltage (see an example in Figure 5a). In this sample, stenabolic was identified, and its spectrum was compared with its corresponding standard (Figure $5 \mathrm{~b}$ ). The obtained spectra, including the chlorine isotope signals, are almost identical, although in the real sample a sodium adduct is observed as well. This could be due to the presence of the SARM as a sodium salt in the sample and/or from excipients not present in the analytical standard. 
Table 1. LDTD-ESI-MS In-Source Fragmentation Tentative Assignments for Studied SARMs

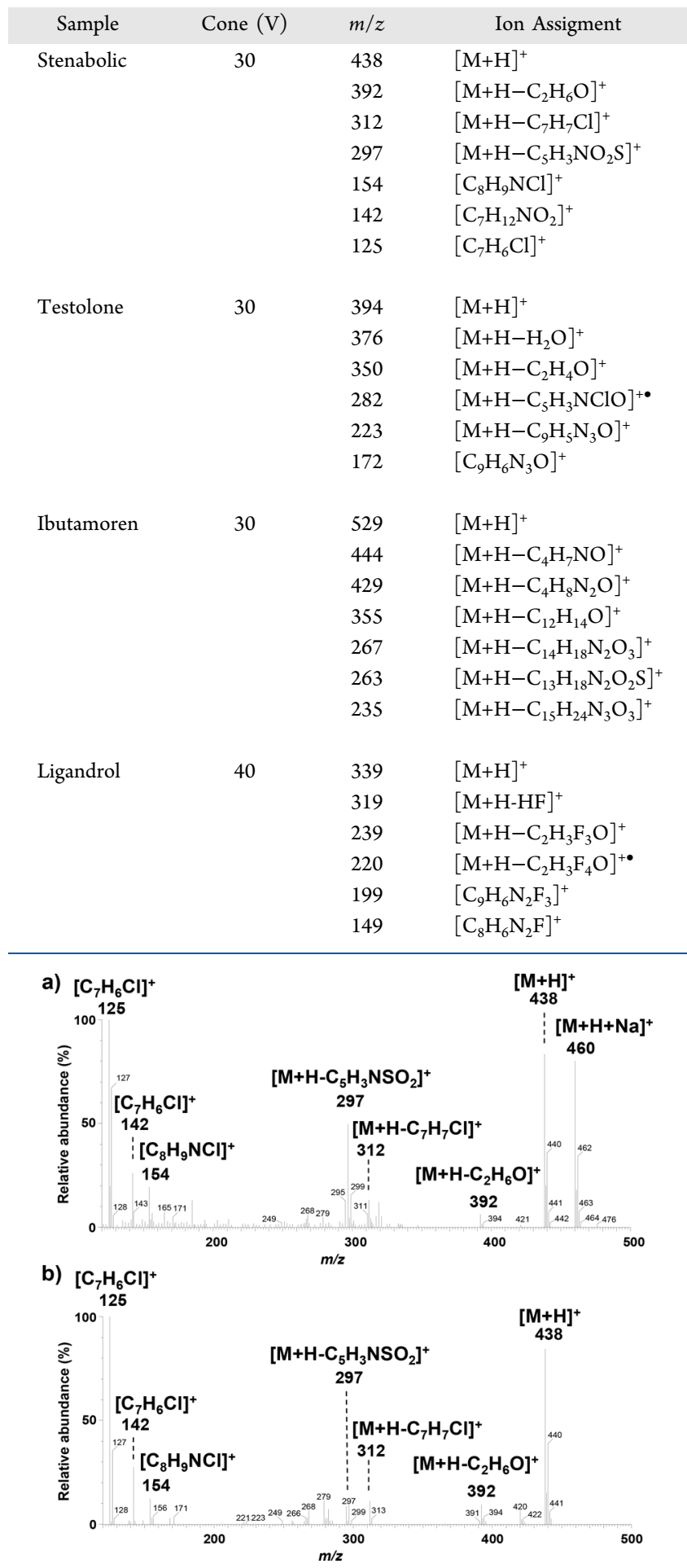

Figure 5. LDTD-ESI-transportable MS full-scan spectrum of (a) sample 1 and (b) stenabolic standard obtained by applying $30 \mathrm{~V}$.

Compared to DART or other plasma sources, the hand-held laser does not require the use of a helium gas supply and additional electricity. Compared to ASAP the described approach is flexible since the laser can be pointed to the surface without any pretreatment. The hand-held laser could be an ideal companion for DESI applications to ablate certain areas of solid samples. As demonstrated in this study, the obtained exact mass or spectra can identify individual compounds; of course, MS/MS capabilities will further contribute to the structure assignments. Moreover, the present setup is compatible with any old or more recent ESI MS system and is, as such, generic.

\section{CONCLUSIONS}

A hand-held diode laser ablation electrospray ionization MS method has been successfully developed to screen solid samples such as powders of illicit drugs rapidly. In contrast to previous rather complex and expensive commercial LDTDESI setups, the present development features a simple inexpensive battery-powered surgical laser diode at $940 \mathrm{~nm}$ to ablate the samples combined with a nano-ESI emitter on either a lab-based HRMS or a simple transportable MS system. The applicability was demonstrated by the identification of SARMs (ostarine, ligandrol, testolone, stenabolic, and ibutamoren) in real samples. Identification of samples can be performed based on in-source fragmentation patterns observed. These initial results demonstrate the applicability of the simplified LDTD-ESI-MS method for future on-site analysis of organic compounds in solid samples.

\section{ASSOCIATED CONTENT}

\section{Supporting Information}

The Supporting Information is available free of charge at https://pubs.acs.org/doi/10.1021/acs.analchem.1c01083.

Table S-1: iLase 10 preprogrammed settings. Table S2: Screening results by LDTD-ESI-HRMS. Figure S-1: Chemical structures and chemical formula of the studied SARMs. Figure S-2: LDTD-ESI-HRMS setup of the laser ablation electrospray source with a batterypowered, hand-held, pseudo-CQ $940 \mathrm{~nm}$ diode laser. Figure S-3: LDTD-ESI-HRMS full scan mass spectra of a standard mixture with ligandrol and stenabolic in positive ion mode and ostarine and andarine in negative ion mode. Figure S-4: Effect of the LDTD-ESI solvent flow rate on the LDTD-ESI-HRMS signal for some representative SARMs. (PDF)

\section{AUTHOR INFORMATION}

\section{Corresponding Author}

Marco H. Blokland - Wageningen Food Safety Research (WFSR), Wageningen University \& Research, $6700 \mathrm{AE}$ Wageningen, The Netherlands; 10 orcid.org/0000-00023751-6065; Phone: +31 031743088;

Email: marco.blokland@wur.nl

\section{Authors}

Ane Arrizabalaga-Larrañaga - Department of Chemical Engineering and Analytical Chemistry, University of

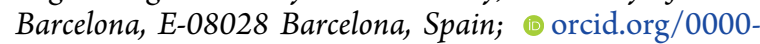
0002-9379-5775

Michel W. F. Nielen - Wageningen Food Safety Research (WFSR), Wageningen University \& Research, $6700 \mathrm{AE}$ Wageningen, The Netherlands; Laboratory of Organic Chemistry, Wageningen University, 6708 WE Wageningen, The Netherlands; (1) orcid.org/0000-0003-4634-0249

Complete contact information is available at: https://pubs.acs.org/10.1021/acs.analchem.1c01083 


\section{Author Contributions}

The manuscript was written through contributions of all authors. All authors have given approval to the final version of the manuscript.

\section{Notes}

The authors declare no competing financial interest.

\section{ACKNOWLEDGMENTS}

Ane Arrizabalaga-Larrañaga thanks the Agency for Management of University and Research Grants (Government of Catalonia) and the European Social Fund for the PhD FIDGR fellowship. This project was financially supported by the Dutch Ministry of Agriculture, Nature and Food Quality (Project KB-23-002-005).

\section{REFERENCES}

(1) de Araujo, W. R.; Cardoso, T. M. G.; da Rocha, R. G.; Santana, M. H. P.; Muñoz, R. A. A.; Richter, E. M.; Paixão, T. R. L. C.; Coltro, W. K. T. Anal. Chim. Acta 2018, 1034, 1-21.

(2) Arrizabalaga-Larrañana, A.; Ayala-Cabrera, J. F.; Sero, R.; Santos, F. J.; Moyano, M. Ambient ionization mass spectrometry in food analysis. In Food Toxicology and Forensics; Galanakis, C. M., Ed.; Elsevier, Inc., 2021; Chapter 9, pp 271-312.

(3) Risoluti, R.; Gullifa, G.; Materazi, S. Front. Chem. 2020, 8, 1-8.

(4) Weesepoel, Y.; Alewijn, M.; Wijtten, M.; Müller-Maatsch, J. J. AOAC Int. 2021, 104, 7-15.

(5) Gentili, S.; Solimini, R.; Tittarelli, R.; Mannocchi, G.; Busardò, F. P. J. Anal. Methods Chem. 2016, 2016, 1234581.

(6) Han, M.; Gong, L.; Wang, J.; Zhang, X.; Jin, Y.; Zhao, R.; Yang,

C.; He, L.; Feng, X.; Chen, Y. Sens. Actuators, B 2019, 292, 94-104.

(7) Liu, J.; Zanardi, S.; Powers, S.; Suman, M. Food Control 2012, 26

(1), 88-91.

(8) Ross, G. M. S.; Salentijn, G. I.; Nielen, M. W. F. Biosensors 2019, 9, 143.

(9) Milman, B. L. TrAC, Trends Anal. Chem. 2015, 69, 24-33.

(10) Takáts, Z.; Wiseman, J. M.; Gologan, B.; Cooks, R. G. Science 2004, 306 (5695), 471-473.

(11) Cody, R. B.; Laramée, J. A.; Durst, H. D. Anal. Chem. 2005, 77 (8), 2297-2302.

(12) Harper, J. D.; Charipar, N. A.; Mulligan, C. C.; Zhang, X.; Cooks, R. G.; Ouyang, Z. Anal. Chem. 2008, 80 (23), 9097-9104.

(13) Nemes, P.; Vertes, A. Anal. Chem. 2007, 79 (21), 8098-8106.

(14) Liu, J.; Wang, H.; Manicke, N. E.; Lin, J. M.; Cooks, R. G.; Ouyang, Z. Anal. Chem. 2010, 82 (6), 2463-2471.

(15) McEwen, C. N.; McKay, R. G.; Larsen, B. S. Anal. Chem. 2005, 77 (23), 7826-7831.

(16) Fiorentin, T. R.; Logan, B. K.; Martin, D. M.; Browne, T.; Rieders, E. F. Forensic Sci. Int. 2020, 313, 110342.

(17) Jager, J.; Gerssen, A.; Pawliszyn, J.; Sterk, S. S.; Nielen, M. W. F.; Blokland, M. H. J. Am. Soc. Mass Spectrom. 2020, 31 (11), 22432249.

(18) Abonamah, J. V.; Eckenrode, B. A.; Moini, M. Forensic Chem. 2019, 16, 100180.

(19) Hu, Q.; Liu, S.; Liu, Y.; Fang, X.; Xu, J.; Chen, X.; Zhu, F.; Ouyang, G. Anal. Chim. Acta 2019, 1050, 88-94.

(20) Blokland, M. H.; Gerssen, A.; Zoontjes, P. W.; Pawliszyn, J.; Nielen, M. W. F. Food Anal. Methods 2020, 13 (3), 706-717.

(21) Cui, Y.; Veryovkin, I. V.; Majeski, M. W.; Cavazos, D. R.; Hanley, L. Anal. Chem. 2015, 87, 367-371.

(22) Vaikkinen, A.; Shrestha, B.; Kauppila, T. J.; Vertes, A.; Kostiainen, R. Anal. Chem. 2012, 84 (3), 1630-1636.

(23) Yung, Y. P.; Wickramasinghe, R.; Vaikkinen, A.; Kauppila, T. J.; Veryovkin, I. V.; Hanley, L. Anal. Chem. 2017, 89 (14), 7297-7301.

(24) Devereaux, Z. J.; Reynolds, C. A.; Fischer, J. L.; Foley, C. D.; DeLeeuw, J. L.; Wager-Miller, J.; Narayan, S. B.; Mackie, K.; Trimpin, S. Anal. Chem. 2016, 88 (22), 10831-10836.

(25) Ding, X.; Liu, K.; Shi, Z. Mass Spectrom. Rev. 2020, 1-40.
(26) Van Geenen, F. A. M. G.; Franssen, M. C. R.; Schotman, A. H. M.; Zuilhof, H.; Nielen, M. W. F. Anal. Chem. 2017, 89 (7), 40314037.

(27) Kostiainen, R.; Kauppila, T. J. J. Chromatogr. A 2009, 1216 (4), 685-699.

(28) Liigand, J.; Laaniste, A.; Kruve, A. J. Am. Soc. Mass Spectrom. 2017, 28 (3), 461-469.

(29) Ventura, E.; Gadaj, A.; Monteith, G.; Ripoche, A.; Healy, J.; Botrè, F.; Sterk, S. S.; Buckley, T.; Mooney, M. H. J. Chromatogr. A 2019, 1600, 183-196.

(30) Snyder, D. T.; Pulliam, C. J.; Ouyang, Z.; Cooks, R. G. Anal. Chem. 2016, 88 (1), 2-29. 